\title{
O EFEITO DA DESATIVAÇÃO TÉRMICA NAS PROPRIEDADES OXIRREDUTORAS E NA ATIVIDADE CATALÍTICA DE CATALISADORES CZ E Pd-CZ
}

Daniela Cruz Damasceno da Silva e Fátima Maria Zanon Zotin*

Departamento de Operações e Projetos Industriais, Instituto de Química, Universidade do Estado do Rio de Janeiro, Rua São Francisco Xavier, 524, 20550-900 Rio de Janeiro - RJ, Brasil

Reiner Neumann

Coordenação de Análises Minerais, Centro de Tecnologia Mineral, Av. Pedro Calmon, 900, Cidade Universitária, 21941-908

Rio de Janeiro - RJ, Brasil

\section{Carla Eponina Hori}

Faculdade de Engenharia Química, Universidade Federal de Uberlândia, Av. João Naves de Ávila, 2121, B1. 1K, Campus Santa Mônica, 38408-100 Uberlândia - MG, Brasil

Mauri José Baldini Cardoso

Centro de Pesquisas e Desenvolvimento Leopoldo Américo Miguez de Melo - PETROBRAS, Av. Horácio Macedo, 950 , Cidade Universitária, 21941-915 Rio de Janeiro - RJ, Brasil

Recebido em 2/2/11; aceito em 22/7/11; publicado na web em 30/9/11

THERMAL AGING EFFECT IN OXI-REDUCTION PROPERTIES AND CATALYTIC ACTIVITY OF CZ AND PD-CZ CATALYST. Automotive catalyst, using in Brazil since 1992, is a essential technology for vehicular emissions control. Noble metals are the active phase of these catalysts, and cerium zirconium mixed oxides (CZ), responsibles for the oxygen storage capacity (OSC), one of the most important aspect for the operational performance of the catalyst. In this context, the oxireduction properties analysis of $\mathrm{CZ}$ and $\mathrm{Pd} / \mathrm{CZ}$ (palladium supported in $\mathrm{CZ}$ ) system are the objective of this study, as well as, the impact of the thermal aging in the OSC. Aging consisted of treatments at 900 or $1200^{\circ} \mathrm{C}$, for 12 or $36 \mathrm{~h}$, in oxidizing condition.

Keywords: cerium-zirconium mixed oxide; thermal aging; automotive catalyst.

\section{INTRODUÇÃO}

Os óxidos mistos de cério e zircônio são componentes essenciais para o bom funcionamento dos catalisadores automotivos comerciais. Um dos principais papéis dos óxidos mistos é a capacidade de estocagem e liberação de oxigênio (OSC), devido à facilidade pela qual os íons cério mudam seu estado de oxidação. A capacidade de estocagem de oxigênio está diretamente relacionada com o par redox $\mathrm{Ce}^{3+} / \mathrm{Ce}^{4+}$. Ao contrário do $\mathrm{CeO}_{2}$ onde a OSC é diretamente proporcional à sua área específica, ${ }^{1}$ nos óxidos mistos de cério e zircônio (CZ) o fenômeno da OSC é volumétrico. Segundo Nagai et al., ${ }^{2}$ que estudaram óxidos mistos de cério e zircônio, durante a liberação de oxigênio o volume do composto aumenta na proporção da mudança do estado de oxidação do $\mathrm{Ce}^{4+}$ (raio iônico $=0,97 \AA$ ) para $\mathrm{Ce}^{3+}$ (raio iônico $=1,14 \AA$ ). A energia de tensionamento que surge desse aumento de volume restringiria qualquer outra mudança de valência do Ce. A introdução de íons zircônio de menor tamanho (raio iônico = 0,84 Å) compensa o incremento de volume, facilitando a mudança de valência.

Com relação às propriedades redox de catalisadores à base de $\mathrm{CZ}$, foi observado que a presença de metais nobres modifica fortemente as características oxirredutoras dos óxidos mistos, devido à ativação do hidrogênio pelo metal e, consequentemente, de sua migração para o suporte, num processo de difusão do hidrogênio conhecido como spillover, favorecendo a redução da superfície do cério em baixas temperaturas. ${ }^{3}$

Um aspecto importante na escolha de metais nobres como candidatos à produção de catalisadores automotivos está associado a não formação de óxidos voláteis. ${ }^{4}$ Estudos têm mostrado que os catalisa-

*e-mail: fmzzotin@gmail.com dores à base de $\mathrm{Pd}$ possuem alta eficiência para a oxidação do $\mathrm{CO}$ e de hidrocarbonetos não convertidos (HC) e moderada atividade para a redução do NO. O Pd apresenta algumas limitações, tais como rápida sinterização sob condições redutoras e envenenamento pelo enxofre em baixas temperaturas. A maior desvantagem da utilização exclusiva do paládio, no entanto, está no fato de se atingir conversões elevadas apenas numa janela estreita de razão ar/combustível. Em contrapartida, o uso do Pd como único componente ativo nos catalisadores automotivos apresenta grande vantagem econômica em relação à platina e ao ródio, devido ao custo e à disponibilidade e, também, à sua alta atividade para as reações de oxidação. ${ }^{5,6}$

Vários trabalhos da literatura se dedicaram ao estudo do envelhecimento térmico de catalisadores automotivos comerciais e seu impacto nas propriedades oxirredutoras. ${ }^{7-16}$ No entanto, nem sempre foi possível estabelecer uma relação direta entre os resultados de redutibilidade e o rigor usado no envelhecimento térmico, entre outros aspectos, devido à complexidade natural do catalisador automotivo que apresenta um grande número de compostos em sua formulação.

Tendo em conta as novas tecnologias onde o catalisador é disposto próximo do motor e, portanto, sendo submetido a altas temperaturas de forma a minimizar as emissões durante o período inicial de operação, fica clara a importância de estudos voltados à desativação térmica para esses sistemas altamente complexos.

Dentro desse contexto e de forma a dar suporte à compreensão do catalisador como um todo, o objetivo deste trabalho foi estudar a evolução da redutibilidade do catalisador $\mathrm{Pd}-\mathrm{CZ}$ e de seu suporte $(\mathrm{CZ})$ com o envelhecimento térmico. O sistema $\mathrm{Pd}-\mathrm{CZ}$ foi escolhido, pois envolve dois dos principais compostos que compõem o catalisador automotivo. Para avaliar o impacto desse envelhecimento na conversão de poluentes, esses catalisadores foram avaliados nas reações de oxidação do $\mathrm{CO}$ e do propano. 


\section{PARTE EXPERIMENTAL}

No preparo do óxido misto de cério e zircônio foi utilizado o método de coprecipitação proposto por Hori et al.. ${ }^{17}$ As quantidades de cada composto foram calculadas de forma a se obter um óxido misto equimolar em cério e zircônio $\left(\mathrm{Ce}_{0,5} \mathrm{Zr}_{0.5} \mathrm{O}_{2}\right)$, aqui denominado CZ. Foram utilizados como precursores nitrato amoniacal de cério $\left(\left(\mathrm{NH}_{4}\right)_{2} \mathrm{Ce}\left(\mathrm{NO}_{3}\right)_{6}\right)$ e nitrato de zirconila $\left(\mathrm{ZrO}\left(\mathrm{NO}_{3}\right)_{2}\right)$, todos SigmaAldrich. Nessa solução, diluída para $0,2 \mathrm{~g} \mathrm{~mol} / \mathrm{L}$ de cério, adicionaramse $90,3 \mathrm{~mL}$ de hidróxido de amônia P.A.Vetec, seguida de agitação magnética vigorosa por $15 \mathrm{~min}$. O precipitado formado foi filtrado a vácuo e calcinado em mufla marca Jung (mod. 0913) em ar estático à $500{ }^{\circ} \mathrm{C}$ por $5 \mathrm{~h}$.

A introdução de $0,5 \%(\mathrm{~m} / \mathrm{m})$ de $\mathrm{Pd}$ no $\mathrm{CZ}$, a partir do cloreto de tetra-amino-paládio II mono-hidratado (Sigma-Aldrich, 98\%) foi feita por impregnação a seco, obtendo-se o catalisador Pd-CZ. A calcinação foi feita em mufla a $500{ }^{\circ} \mathrm{C}$ por $5 \mathrm{~h}$, a uma taxa de aquecimento de $10{ }^{\circ} \mathrm{C} / \mathrm{min}$.

Parte do catalisador e suporte preparados foram submetidos a envelhecimento térmico em mufla a duas temperaturas, 900 e 1200 ${ }^{\circ} \mathrm{C}$, por 12 e $36 \mathrm{~h}$, resultando nas amostras CZ-900-12 h, CZ-900-36 h, CZ-1200-12 h, CZ-1200-36 h, Pd-CZ-900-12 h, Pd-CZ-900-36 h e Pd-CZ-1200-12 h. A amostra Pd-CZ-1200-36 h foi descartada, pois sofreu fusão durante o envelhecimento.

As amostras foram analisadas quanto ao teor de cério, zircônio e paládio pela técnica de espectrometria de emissão óptica com plasma indutivamente acoplado (ICP-OES), marca Horiba, Jobin Yvon Inc.

As determinações da área específica e das isotermas de adsorção das amostras novas e envelhecidas foram feitas em um equipamento ASAP, modelo 2020, da Micromeritics. As amostras foram submetidas a um pré-tratamento que consistiu em aquecer a amostra a $300^{\circ} \mathrm{C} \mathrm{sob}$ vácuo de 50 mTorr por $2 \mathrm{~h}$.

Ensaios de análise térmica das amostras CZ e Pd-CZ novas foram realizados em um equipamento TG/DTA, modelo SDT 2960, TA Intruments. As análises foram realizadas sob ar estático, na faixa de temperatura entre a ambiente e $1200^{\circ} \mathrm{C}$, usando taxa de aquecimento de $10^{\circ} \mathrm{C} / \mathrm{min}$.

Nas análises de difração de raios X (DRX) usou-se o método do pó e utilizou-se um equipamento Rigaku Miniflex II com espelho de Goeble, com radiação $\mathrm{Cu} \mathrm{K} \alpha(40 \mathrm{kV} / 30 \mathrm{~mA})$, velocidade do goniômetro de $0,060^{\circ}$ por passo e tempo por passo de $2 \mathrm{~s}$ por ponto.

Nos ensaios de redução à temperatura programada foi utilizada uma unidade multipropósito constituída de um reator de quartzo tipo "tubo em U" e de um forno vertical com controle de temperatura do tipo PID. No pré-tratamento, amostras de $250 \mathrm{mg}$ com granulometria na faixa de 40-60 mesh foram reduzidas por um fluxo de composição $1,61 \% \mathrm{H}_{2} / \mathrm{Ar}$ à taxa de $10{ }^{\circ} \mathrm{C} / \mathrm{min}$ até $500{ }^{\circ} \mathrm{C}$, com patamar de $1 \mathrm{~h}$; posteriormente, a amostra foi reoxidada por um fluxo de composição $5 \% \mathrm{O}_{2} / \mathrm{He}$, nas mesmas condições. Após o resfriamento do reator (temperatura ambiente), foi iniciada a TPR com a mistura $\mathrm{H}_{2} / \mathrm{Ar}$ utilizada inicialmente, também sob taxa de aquecimento de $10^{\circ} \mathrm{C} / \mathrm{min}$ e patamar de $1 \mathrm{~h}$ a $500^{\circ} \mathrm{C}$. Os gases foram fornecidos pela Linde gases.

$\mathrm{Na}$ avaliação catalítica foram estudadas duas reações: a oxidação do CO e do propano, usando a mistura MPP1 (1,0\% de CO, 0,33\% de $\mathrm{H}_{2}, 1,27 \%$ de $\mathrm{O}_{2}, 9,3 \%$ de $\mathrm{CO}_{2}$ e $0,12 \%$ de $\mathrm{C}_{3} \mathrm{H}_{8}$, balanço em $\mathrm{He}$ ). A unidade de avaliação catalítica é constituída de um reator de vidro pirex tipo "tubo em U", de diâmetro interno de $0,66 \mathrm{~cm}$, um forno vertical com controle de temperatura. A unidade é acoplada, em linha, a um cromatógrafo gasoso Agilent $6890 \mathrm{~N}$, com duas colunas empacotadas, uma Porapak N (Supelco) e uma peneira molecular 13 X (Supelco) 45/60. Nos testes catalíticos utilizaram-se $120 \mathrm{mg}$ de amostras em pó, com granulometria entre 40 e 60 mesh. Efeitos térmicos (hot-spots) foram minimizados diluindo o catalisador em carbeto de silício numa proporção mássica de 1:3. Foi feito um pré- tratamento em fluxo de $\mathrm{He}$, a $150{ }^{\circ} \mathrm{C}$ por $1 \mathrm{~h}$, a $10{ }^{\circ} \mathrm{C} / \mathrm{min}$. O teste catalítico foi realizado usando rampa de temperatura de $2{ }^{\circ} \mathrm{C} / \mathrm{min}$ até $500{ }^{\circ} \mathrm{C}$, temperatura esta que foi mantida constante por mais $1 \mathrm{~h}$. A vazão em todos os testes foi mantida em $13,5 \mathrm{~L} / \mathrm{h}$.

\section{RESULTADOS E DISCUSSÃO}

Os resultados de análise química confirmaram a presença equimolar de cério e zircônio no óxido misto $\mathrm{CZ}$ e a presença de $0,52 \%$ $(\mathrm{m} / \mathrm{m})$ de Pd no óxido misto Pd-CZ.

Os resultados de área específica para os óxidos mistos CZe Pd-CZ estão reunidos na Tabela 1. Observa-se que a impregnação de paládio no $\mathrm{CZ}$ ocasionou uma ligeira queda no valor da área específica. Em termos texturais, a presença de $\mathrm{Pd}$ praticamente não promoveu mudanças no CZ. No entanto, nos catalisadores envelhecidos termicamente as diferenças são significativas.

Tabela 1. Propriedades texturais e tamanho de partícula dos óxidos mistos $\mathrm{CZ}$ e Pd-CZ novos e envelhecidos

\begin{tabular}{lccccc}
\hline Amostra & $\begin{array}{c}\text { Área } \\
\text { específica } \\
\left(\mathrm{m}^{2} / \mathrm{g}\right)\end{array}$ & $\begin{array}{c}\text { Diâmetro } \\
\text { de poro } \\
(\AA)\end{array}$ & $\begin{array}{c}\text { Volume } \\
\text { de poro } \\
\left(\mathrm{cm}^{3} / \mathrm{g}\right)\end{array}$ & $2 \theta\left(^{\circ}\right)$ & $\mathrm{D}(\mathrm{nm})$ \\
\hline $\mathrm{CZ}$ & 93 & 60 & 0,16 & 29,2 & 5,4 \\
$\mathrm{CZ}-900-12 \mathrm{~h}$ & 25 & 182 & 0,10 & 29,2 & 10,4 \\
$\mathrm{CZ}-900-36 \mathrm{~h}$ & 20 & 227 & 0,10 & 29,2 & 9,8 \\
$\mathrm{CZ}-1200-12 \mathrm{~h}$ & 0,2 & $*$ & $*$ & 28,9 & 30,2 \\
$\mathrm{CZ}-1200-36 \mathrm{~h}$ & $*$ & $*$ & $*$ & 28,9 & 30,2 \\
Pd-CZ & 84 & 77 & 0,17 & 29,2 & 6,0 \\
Pd-CZ-900-12 h & 23 & 201 & 0,13 & 29,2 & 11,0 \\
Pd-CZ-900-36 h & 19 & 201 & 0,11 & 29,2 & 9,6 \\
Pd-CZ-1200-12 h & $*$ & $*$ & $*$ & 28,9 & 29,8 \\
\hline
\end{tabular}

* não detectado.

A Figura 1 apresenta as isotermas de adsorção dos catalisadores $\mathrm{CZ}$, novos e envelhecidos. Nota-se que o envelhecimento do $\mathrm{CZ}$ a $900{ }^{\circ} \mathrm{C}$ por 12 ou $36 \mathrm{~h}$ deslocou significativamente as isotermas em relação ao $\mathrm{CZ}$ novo. Também se destaca que foram obtidas as isotermas das amostras envelhecidas a $1200{ }^{\circ} \mathrm{C}$. No caso dos catalisadores Pd-CZ o mesmo ocorreu, porém não foi possível obter as isotermas das amostras submetidas a tratamento térmico a $1200{ }^{\circ} \mathrm{C}$ por 12 ou 36 h. É possível que a presença de paládio esteja promovendo a sinterização do CZ.

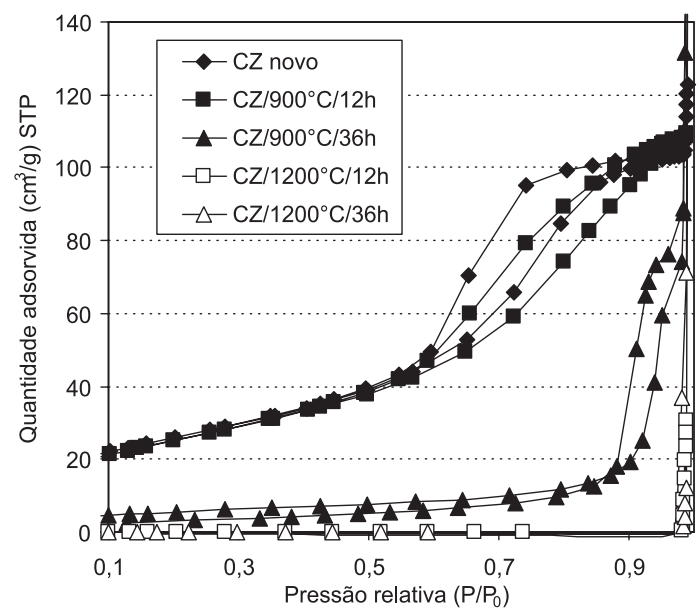

Figura 1. Isotermas dos catalisadores da série CZ 
Segundo a classificação da IUPAC, ${ }^{18}$ para as séries CZ e Pd-CZ, as isotermas são do tipo IV (típica de sólidos mesoporosos) no caso dos catalisadores novos. Para a maioria das amostras envelhecidas a 900 e $1200^{\circ} \mathrm{C}$ elas são do tipo II (característica de sólidos não porosos ou macroporos). A exceção é a amostra CZ-900-12 que ainda mantém a isoterma tipo IV, mas já mostra deslocamento em relação à observada no caso do $\mathrm{CZ}$ novo (Figura 1). Aparentemente, a mudança da isoterma para o tipo II deve-se ao crescimento das partículas devido ao processo de sinterização. As isotermas relativas às amostras $\mathrm{Pd}-\mathrm{CZ}$ seguem o mesmo padrão das observadas para os catalisadores $\mathrm{CZ}$.

Assim, o envelhecimento acarreta uma evolução progressiva na isoterma, fenômeno este também observado por Vidal et al., ${ }^{19}$ no estudo do comportamento redox de óxidos mistos de $\mathrm{CeO}_{2}-\mathrm{ZrO}_{2}$ após a realização de TPR a $1000{ }^{\circ} \mathrm{C}$. Todas essas mudanças são atribuídas às alterações na distribuição de tamanho de poros, que se caracteriza pelo deslocamento para diâmetros de poros maiores, conforme se pode observar na Tabela 1, para ambos, CZ e Pd-CZ. Nota-se, como esperado, que foram observadas diferenças importantes na distribuição de tamanho de poros quando se comparam os catalisadores novos com os submetidos a envelhecimento térmico. Entre os catalisadores envelhecidos as diferenças foram pequenas.

Os resultados de difração de raios X da série CZ são apresentados na Figura 2. A identificação dos picos foi feita usando-se o software XRD Evolution Program (EVA). Para as amostras CZ, CZ-900-12 h e CZ-900-36 h, confirma-se a presença do óxido misto $\mathrm{Ce}_{0,5} \mathrm{Zr}_{0,5} \mathrm{O}_{2}$. O envelhecimento a $1200{ }^{\circ} \mathrm{C}$ promoveu o fenômeno de segregação de fases (demixing) da amostra com, pelo menos, a formação de dois óxidos mistos diferentes, um rico em cério, de estrutura cúbica $(\mathrm{C})$, e o outro rico em zircônio, de estrutura tetragonal ( $\mathrm{T}$ ); cujas composições são $\mathrm{Ce}_{0,75} \mathrm{Zr}_{0,25} \mathrm{O}_{2}$ e $\mathrm{Ce}_{0,16} \mathrm{Zr}_{0,84} \mathrm{O}_{2}$, respectivamente, também identificados por difração de raios X. Esses resultados estão de acordo com os de Bozo et al. ${ }^{11}$ que estudaram óxidos mistos de $\mathrm{Ce}$ e $\mathrm{Zr}$ envelhecidos hidrotermicamente. Segundo os autores, a posição da linha principal de difração da fase tetragonal não varia e corresponde à fase $\mathrm{Ce}_{0,16} \mathrm{Zr}_{0,84} \mathrm{O}_{2}$ (JCPDS-ICDD data base (file no. 38-1437).

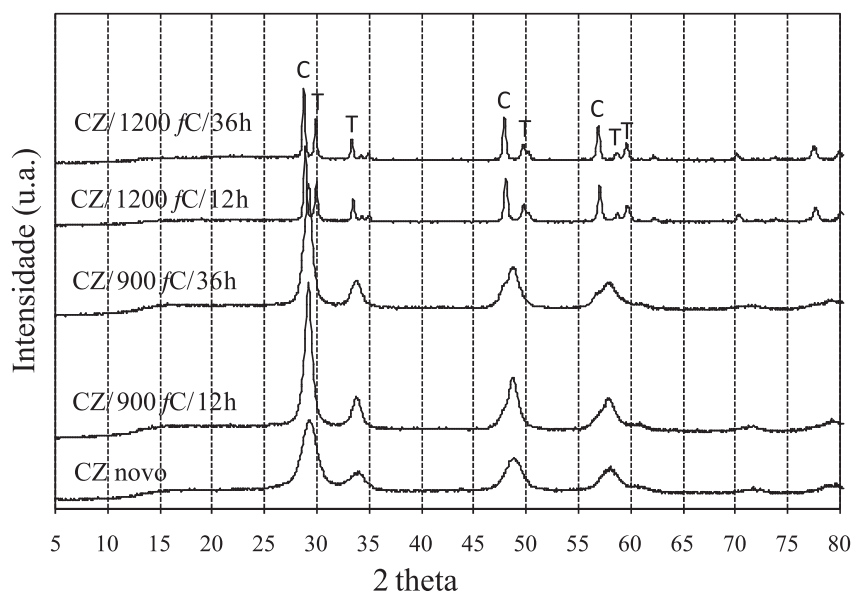

Figura 2. Distribuição do volume de poros dos catalisadores da série CZ

No caso da série Pd-CZ, para o catalisador novo e para o PdCZ900-12 h, não se observou a ocorrência de demixing, nem a formação de fases extras em relação à presença de paládio, o que é razoável considerando que foram adicionados teores relativamente baixos de $\operatorname{Pd}(0,52 \% \mathrm{~m} / \mathrm{m})$. Já, para as amostras termicamente mais envelhecidas (Pd-CZ-900-36 h e Pd-CZ-1200-12 h) pode-se observar ligeiro indício da presença de $\mathrm{PdO}$, provavelmente associado à aglomeração promovida pelas altas temperaturas. O difratograma da amostra Pd-CZ-1200 $12 \mathrm{~h}$ indica a ocorrência da segregação de fases também identificadas como
$\mathrm{Ce}_{0,75} \mathrm{ZrO}_{, 25} \mathrm{O}_{2}$ e $\mathrm{Ce}_{0,16} \mathrm{Zr}_{0,84} \mathrm{O}_{2}$, como também observado para $\mathrm{CZ}-1200$ $12 \mathrm{~h}$ e CZ-1200-36 h.

Ainda com base nos resultados de DRX, foi obtido o diâmetro de partículas do óxido misto de cério-zircônio usando a Lei de Scherrer, como pode ser observado na Tabela 1 . Nota-se que os resultados são coerentes, indicando um aumento no tamanho de partícula com o aumento da temperatura de envelhecimento. Também se observa que o tempo de envelhecimento numa dada temperatura praticamente não altera o diâmetro das partículas.

Um experimento de análise térmica (em ar, até $1200^{\circ} \mathrm{C}$ ) também foi realizado na amostra $\mathrm{CZ}$ com o intuito de se acompanhar as alterações de fases observadas (Figura 3). O primeiro pico endotérmico é observado a $65^{\circ} \mathrm{C}$ e, provavelmente, se deve à eliminação de água fisissorvida. Em seguida, iniciou-se a formação de um largo pico endotérmico, que atinge o ápice em $1079{ }^{\circ} \mathrm{C}$, atribuído ao fenômeno de segregação de fases. Este resultado está coerente com os de DRX deste trabalho onde esse fenômeno apenas é observado após envelhecimento a $1200{ }^{\circ} \mathrm{C}$.

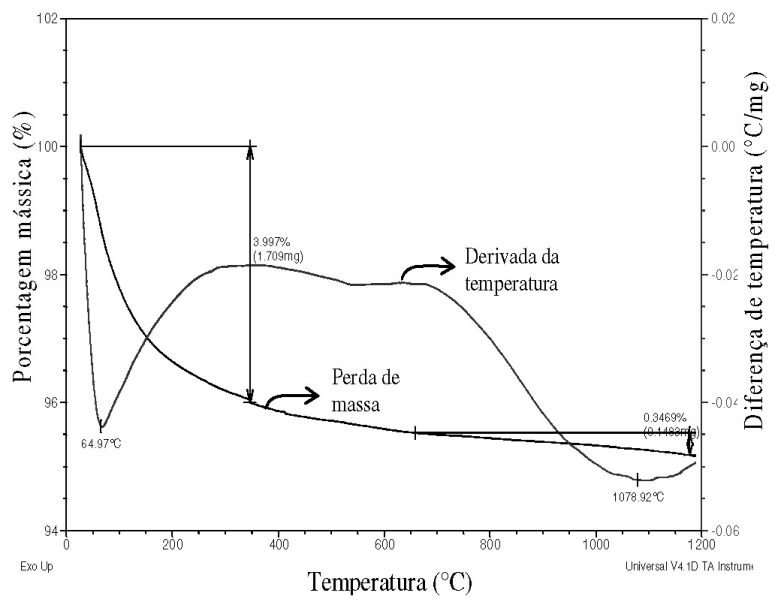

Figura 3. Análise térmica do catalisador CZ novo

As Figuras 4 e 5 apresentam os perfis de redução a temperatura programada obtidos para as amostras das séries CZ e Pd-CZ, respectivamente.

Na Figura 4, observa-se que a amostra de $\mathrm{CZ}$ novo começou a apresentar uma redução significativa a partir de $300^{\circ} \mathrm{C}$. Os resultados para a amostra CZ-900-12 h indicaram uma perda na capacidade de redução da amostra, o que pode estar associado a uma diminuição da área específica que passou de 93 para $25 \mathrm{~m}^{2} / \mathrm{g}$. No caso da amostra CZ-900-36 h, no entanto, observa-se que sua redução ocorre em temperaturas menores. Assim, $24 \mathrm{~h}$ adicionais a $900{ }^{\circ} \mathrm{C}$ que pouco alteraram o valor da área específica (de 25 para $20 \mathrm{~m}^{2} / \mathrm{g}$ ), promoveram alterações significativas nas propriedades oxirredutoras do óxido misto de Ce e Zr. Os resultados de DRX não indicaram alterações estruturais perceptíveis entre as amostras CZ-900-12 h e CZ-900-36 h. Entretanto, acredita-se que mudanças físico-químicas devam estar ocorrendo gerando espécies mais facilmente redutíveis, mas que não são nitidamente visíveis por DRX. Wu et al.,$^{20}$ que estudaram o óxido misto $\mathrm{Ce}_{0,67} \mathrm{Zr}_{0,33} \mathrm{O}_{2}$ novo e envelhecido a $950{ }^{\circ} \mathrm{C}$ por $20 \mathrm{~h} \mathrm{em}$ ar estático, também observaram melhora nas propriedades oxirredutoras após envelhecimento térmico. Fornasiero et al. ${ }^{21}$ no estudo de catalisadores $\mathrm{Ce}_{0,5} \mathrm{Zr}_{0,5} \mathrm{O}_{2}$, verificaram o mesmo efeito após ciclos de redução e oxidação a $1000{ }^{\circ} \mathrm{C}$. Para a amostra nova foi observado um comportamento muito similar ao indicado na Figura 4, com o aumento do número de ciclos de redução/oxidação houve um deslocamento dos picos para temperaturas mais baixas. Provavelmente esse comportamento se deve a um rearranjo suave dos átomos em 
relação à sua posição original tendendo a uma estrutura mais aberta, proporcionado uma maior mobilidade dos ânions do bulk.

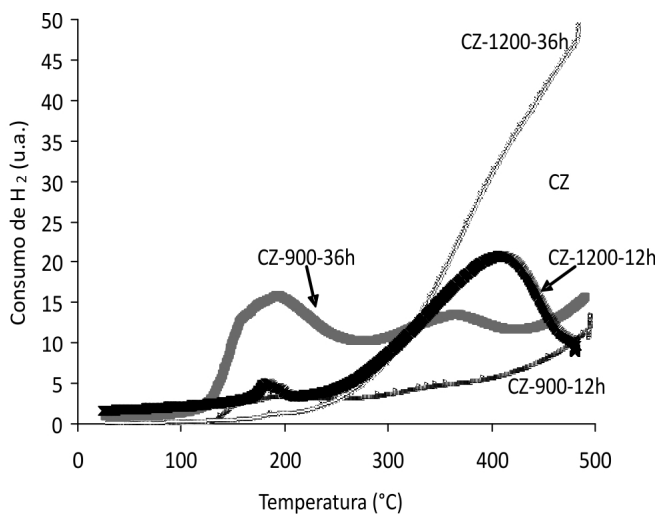

Figura 4. Perfis de TPR dos catalisadores da série CZ

Apesar de apresentarem áreas específicas desprezíveis, as amostras CZ-1200-12 h e CZ-1200-36 h mostraram uma maior redutibilidade quando comparadas à amostra de $\mathrm{CZ}$ novo, em termos de temperatura de início da redução, porém a amostra CZ-900-36 h se reduziu em temperaturas ainda mais baixas. Apesar da amostra CZ-1200-36 h se reduzir em temperaturas elevadas, o envelhecimento a $1200{ }^{\circ} \mathrm{C}$ por $36 \mathrm{~h}$ proporcionou uma redução quantitativamente maior como pode ser verificado pela Tabela 2, que apresenta, para cada catalisador, os resultados do consumo de hidrogênio em $\mu$ mols de $\mathrm{H}_{2} / g$ catalisador. Esse cálculo foi feito com base na integração das curvas de RTP por meio do software Microcal Origin. Esses resultados estão de acordo com os obtidos por Fally et al., ${ }^{22}$ que estudaram óxidos mistos $\mathrm{CeO}_{2}$ $\mathrm{ZrO}_{2}$ de alta área específica, submetidos a envelhecimentos entre 900 e $950^{\circ} \mathrm{C}$. Segundo esses autores, os óxidos mistos após ciclos redox promovem a redução a baixa temperatura, com essa melhora sendo mais significativa para amostras envelhecidas de baixa área. Além disso, a influência da área não contribui com esse fenômeno, mas sim as condições de preparo e ativação do CZ.

Tabela 2. Consumo de hidrogênio por grama de catalisador, obtido a partir dos resultados de TPR

\begin{tabular}{lccc}
\hline Amostra & $\begin{array}{c}\text { Consumo de } \mathrm{H}_{2} \\
\left(\mu \mathrm{mols} \mathrm{H}_{2} / \mathrm{g}\right)\end{array}$ & Amostra & $\begin{array}{c}\text { Consumo de } \mathrm{H}_{2} \\
\left(\mu \mathrm{mols} \mathrm{H}_{2} / \mathrm{g}\right)\end{array}$ \\
\hline $\mathrm{CZ}$ & $170(5 \%)$ & Pd-CZ & $749(21 \%)$ \\
$\mathrm{CZ}-900-12 \mathrm{~h}$ & $58(1,7 \%)$ & Pd-CZ-900-12h & $339(8,5 \%)$ \\
$\mathrm{CZ}-900-36 \mathrm{~h}$ & $211(6,2 \%)$ & Pd-CZ-900-36h & $237(5,5 \%)$ \\
$\mathrm{CZ}-1200-12 \mathrm{~h}$ & $106(3,1 \%)$ & Pd-CZ-1200-12h & $*$ \\
$\mathrm{CZ}-1200-36 \mathrm{~h}$ & $330(9,7 \%)$ & & \\
\hline
\end{tabular}

*não detectado. \% entre parêntesis: percentual de redução do CZ.

Os perfis de TPR das amostras Pd-CZ, novas e envelhecidas, estão apresentados na Figura 5. Para o catalisador novo e para os envelhecidos a $900{ }^{\circ} \mathrm{C}$ observa-se a formação de um único e largo pico de consumo de $\mathrm{H}_{2}$ a baixa temperatura (até, aproximadamente, $200{ }^{\circ} \mathrm{C}$ ). Lambrou et al. ${ }^{23}$ estudando a capacidade de estocagem de oxigênio de catalisadores comerciais $\mathrm{Pd} / \mathrm{Rh}$, verificaram na TPR a formação de dois picos a baixas temperaturas, relacionados à redução dos metais. Segundo os autores, é possível estar também incluída a redução concomitante de parte do $\mathrm{CZ}$.

Neste trabalho, para o catalisador Pd-CZ, Figura 5, observa-se um intenso e largo pico com máximo ao redor de $130^{\circ} \mathrm{C}$. Esse fenômeno foi atribuído à habilidade do paládio em ativar o $\mathrm{H}_{2} \mathrm{e}$ "espalhá-lo" sobre o suporte, facilitando sua redução (spillover de $\mathrm{H}_{2}$ ). Assim, a impregnação de paládio no óxido misto modificou fortemente o comportamento oxirredutor do mesmo e promoveu a redução do suporte (CZ), levando a um elevado consumo de hidrogênio. Sem o metal, a ativação do hidrogênio é dificultada e torna-se a etapa cinética controladora da reação. ${ }^{3,20}$

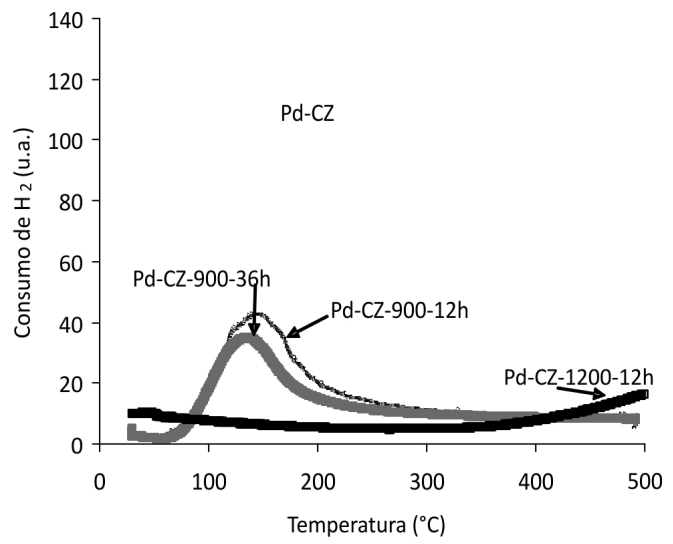

Figura 5. Perfis de TPR dos catalisadores da série Pd-CZ

Na Tabela 2 também se apresentam os resultados de consumo de $\mathrm{H}_{2}$ por grama de catalisador durante a TPR, bem como o percentual de redução do CZ. No caso do catalisador CZ novo, o consumo foi de $170 \mu \mathrm{mols} \mathrm{H}_{2} / \mathrm{g}$, o que equivale a $5 \%$ de redução do CZ, aproximadamente. Já para o Pd-CZ foram consumidos $749 \mu$ mols de $\mathrm{H}_{2} / g$ cat. A quantidade de paládio no catalisador Pd-CZ é de $0,52 \%(\mathrm{~m} / \mathrm{m})$ o que corresponde a um consumo de $49 \mu \mathrm{mols}$ de $\mathrm{H}_{2} / \mathrm{g}$ para sua redução. $\mathrm{O}$ restante estaria associado à redução do óxido misto, ou seja, aproximadamente $21 \%$ do $\mathrm{CZ}$ foram reduzidos, obtendo-se um aumento considerável na redução do CZ. É importante esclarecer que a estimativa do percentual de redução está admitindo que todo o paládio está sendo reduzido, o que pode ser questionado, uma vez que a aglomeração pode resultar numa área metálica menor.

Após o envelhecimento a $900{ }^{\circ} \mathrm{C}$ do $\mathrm{Pd}-\mathrm{CZ}$, nota-se um ligeiro deslocamento dos picos de redução para temperaturas mais altas, além de uma diminuição expressiva do consumo de $\mathrm{H}_{2}$. Wu et al. ${ }^{20}$ observaram comportamento semelhante no estudo de catalisadores à base de $\mathrm{Ce}_{0,67} \mathrm{Zr}_{0,33} \mathrm{O}_{2}$ impregnados com Pt. Segundo eles, o pico de redução do $\mathrm{Ce}_{0,67} \mathrm{Zr}_{0,33} \mathrm{O}_{2}$ após o envelhecimento a $900{ }^{\circ} \mathrm{C}$ por $20 \mathrm{~h}$, além de se deslocar para temperaturas maiores, também apresentou queda drástica em sua intensidade, indicando diminuição no consumo de hidrogênio provavelmente relacionada à perda de área metálica, que deixa de promover a redução do suporte.

Os resultados de atividade catalítica em termos de conversões de $\mathrm{CO}$ e propano (mistura MPP1), das séries de catalisadores $\mathrm{CZ}$ e Pd-CZ são apresentados através de curvas de lightoff nas Figuras 6 e 7 , respectivamente.

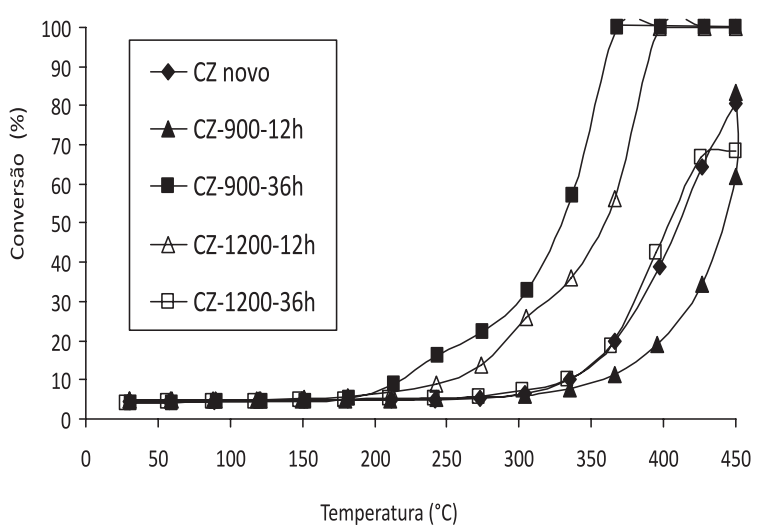

Figura 6. Conversão de CO e de propano para os catalisadores da série CZ 
$\mathrm{Na}$ oxidação do CO, nota-se que as amostras CZ-900-36 h e CZ-1200-12 h alcançaram conversões de $100 \%$ em temperaturas superiores a $350^{\circ} \mathrm{C}$. O catalisador $\mathrm{CZ}$ novo não se mostrou tão ativo, atingindo ao redor de $80 \%$ de conversão a $450{ }^{\circ} \mathrm{C}$. Assim, curiosamente, o catalisador novo apresentou um desempenho pior do que aqueles envelhecidos a $1200{ }^{\circ} \mathrm{C}$, apesar de todos os catalisadores da série CZ-1200 apresentarem uma área específica praticamente nula. Por outro lado, esses resultados estão coerentes com os de RTP, onde se observa que o catalisador CZ-900-36 h apresenta picos de consumo de $\mathrm{H}_{2}$ em temperaturas mais baixas, indicando ser mais facilmente reduzido, seguido pelo CZ-1200-12 h e assim, sucessivamente.

As reações que provavelmente ocorrem são:

$$
\begin{aligned}
& \mathrm{CO}+2 \mathrm{CeO}_{2} \rightarrow \mathrm{CO}_{2}+\mathrm{Ce}_{2} \mathrm{O}_{3} \\
& \mathrm{Ce}_{2} \mathrm{O}_{3}+\frac{1}{2} \mathrm{O}_{2} \rightarrow 2 \mathrm{CeO}_{2}
\end{aligned}
$$

Observa-se que o envelhecimento térmico, seja a 900, seja a $1200{ }^{\circ} \mathrm{C}$, situação em que se tem sinterização intensa, mostrou promover a oxidação do CO. A exceção foi o CZ-900-12 h, que apresentou um desempenho pior do que o novo.

Isso mostra que a reação de oxidação do CO ocorre essencialmente pelo mecanismo de oxirredução do óxido misto. Provavelmente, como nas análises de TPR, dependendo da temperatura e do tempo de exposição, a sinterização do óxido misto induz a uma modificação estrutural que promove o ciclo redox $\mathrm{Ce}^{4+} \leftrightarrow \mathrm{Ce}^{3+}$. A explicação pode estar na estrutura do $\mathrm{CZ}$, onde a presença de $\mathrm{Zr}$ promove defeitos estruturais que aparecem em maior quantidade após um tratamento térmico "controlado", onde se varia a temperatura e o tempo de envelhecimento. Esses novos sítios explicariam as propriedades únicas associadas à sua maior redutibilidade.

Nesse sentido, Kaspar et al., ${ }^{24}$ que estudaram o efeito de tratamentos térmicos entre $600 \mathrm{e} 1000^{\circ} \mathrm{C} \mathrm{em} \mathrm{CeO}_{2}-\mathrm{ZrO}_{2}$, verificaram que o processo de sinterização modifica significativamente a estrutura do óxido misto através da criação de um sólido com defeitos. Esses defeitos estruturais têm sido apontados como peça fundamental nas propriedades catalíticas de materiais à base de $\mathrm{CeO}_{2}$. Assim, a oxidação do $\mathrm{CO}$ não se correlacionou com as propriedades texturais dos catalisadores, o que está de acordo com o fato da OSC dos catalisadores CZ estar associada a um processo volumétrico e não superficial. ${ }^{2}$

Neste trabalho, a questão da sinterização foi abordada usando temperaturas mais elevadas (até $1200^{\circ} \mathrm{C}$ ) e pelos resultados obtidos (Figura 6), as considerações de Kaspar et al. ${ }^{24}$ ainda se aplicam. Vale destacar que mesmo após a ocorrência de segregação de fases (amostra calcinada a $1200^{\circ} \mathrm{C}$ por $12 \mathrm{~h}$ ) essa ativação é observada, ou seja, mesmo com uma mistura física das fases segregadas e uma área específica praticamente nula, elas ainda apresentaram propriedades para promover a oxidação do $\mathrm{CO}$, com conversões superiores ou próximas às obtidas para o $\mathrm{CZ}$ novo. Salienta-se ainda que a amostra CZ-900-12 h foi a que mostrou pior conversão, o que também está coerente com os resultados de TPR, apresentando menor redução entre as amostras da série $\mathrm{CZ}$.

Com relação às curvas de lightoff para a conversão de propano, ao contrário do observado para o $\mathrm{CO}$, tem-se uma atividade muito baixa, próxima de zero para a maioria dos catalisadores nessa reação. A única exceção foi o $\mathrm{CZ}$, que mostrou pequena atividade a partir de $350^{\circ} \mathrm{C}$, alcançando $15 \%$ a $450{ }^{\circ} \mathrm{C}$. Assim, a oxidação do propano não ocorre através de um mecanismo de oxirredução. Segundo Burch et al. ${ }^{25}$ é preciso ativar a ligação $\mathrm{C}-\mathrm{H}$ dos hidrocarbonetos na superfície de um catalisador metálico a fim de que sejam oxidados. Portanto, a presença do metal nobre é imprescindível para a conversão do propano.

A Figura 7 apresenta as curvas de lightoff para a conversão de $\mathrm{CO}$ e do propano, para as séries Pd-CZ, usando a mistura MPP1.
Conforme o esperado, a atividade dos catalisadores aumentou significativamente em relação aos da série $\mathrm{CZ}$, tanto em termos de

\begin{tabular}{|c|c|}
\hline $\begin{array}{l}\rightarrow \mathrm{Pd}-\mathrm{CZ}(\mathrm{CO}) \\
\triangle \mathrm{Pd}-\mathrm{CZ}-900-12 \mathrm{~h}(\mathrm{CO}) \\
\backsim-\mathrm{Pd}-\mathrm{CZ}-900-12 \mathrm{~h}(\mathrm{C} 3 \mathrm{H} 8) \\
\rightarrow-\mathrm{Pd}-\mathrm{CZ}-1200-12 \mathrm{~h}(\mathrm{CO})\end{array}$ & $\begin{array}{l}- \text { - Pd-CZ-900-36h (CO) } \\
\diamond-\mathrm{Pd}-\mathrm{CZ}(\mathrm{C} 3 \mathrm{H} 8) \\
\square-\mathrm{Pd}-\mathrm{CZ}-900-36 \mathrm{~h}(\mathrm{C} 3 \mathrm{H} 8) \\
-\mathrm{Pd}-\mathrm{CZ}-1200-12 \mathrm{~h}(\mathrm{C} 3 \mathrm{H} 8)\end{array}$ \\
\hline
\end{tabular}
conversão de $\mathrm{CO}$ como de propano.

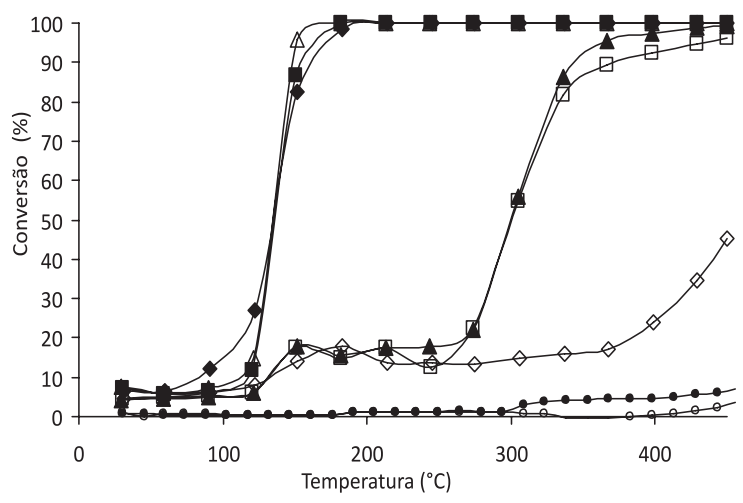

Figura 7. Conversão de CO e de propano para os catalisadores da série $\mathrm{Pd}-\mathrm{CZ}$

Na oxidação do $\mathrm{CO}$ a presença de Pd eliminou o efeito do tempo de envelhecimento a $900{ }^{\circ} \mathrm{C}$, já que as curvas de lightoff dos catalisadores Pd-CZ, Pd-CZ-900-12 h e Pd-CZ-900-36 h praticamente coincidiram.

Sabe-se que a interação entre o paládio e o $\mathrm{CZ}$ pode promover a oxidação do $\mathrm{CO}$, principalmente porque gera um caminho reacional de menor energia de ativação no qual oxigênios interfaciais atuariam como espécies ativas. No caso, é provável que o metal promova no óxido um aumento na concentração de equilíbrio de vacâncias de oxigênio, ou seja, ocorre a transferência de elétrons do óxido para o metal nobre, resultando na diminuição da energia de ativação para a formação de vacâncias de oxigênio. ${ }^{11}$ Desta forma, a presença do metal nobre amplia o número de vacâncias de oxigênio e aumenta a mobilidade dos oxigênios, consequentemente melhorando a atividade catalítica.

O envelhecimento do catalisador $\mathrm{Pd}-\mathrm{CZ}$ por $12 \mathrm{~h}$ a $1200{ }^{\circ} \mathrm{C}$ desativou-o por completo. Estudos demonstraram que a perda da atividade catalítica está relacionada com a perda da área superficial, que é marcante para este catalisador. Além disso, a exposição a temperaturas severas causa mudanças nas fases superficiais ativas e nos estados químicos dos metais ativos, no caso o Pd, o que afeta a atividade catalítica. ${ }^{9}$ Com a sinterização do paládio decresce o número de centros ativos em sua superfície, e uma sinterização conjunta do CZ e do paládio diminui o número de interações entre ambos. ${ }^{5}$ Dessa forma, considerando a atividade do suporte $\mathrm{CZ}$ antes do envelhecimento a $1200{ }^{\circ} \mathrm{C}$ (Figura 7), aparentemente o paládio catalisou a sinterização do suporte, desativando-o. Esses resultados estão de acordo com os de RTP onde não se observou praticamente redução das amostras envelhecidas a $1200{ }^{\circ} \mathrm{C}$ (Figura 6), diferente do que se observa para as amostras $\mathrm{CZ}$, onde esse mesmo envelhecimento aumenta significativamente a redutibilidade das mesmas (Figura 5).

A Figura 7 também apresenta as curvas de lightoff de conversão do propano. A atividade para essa reação, conforme já discutido, não está associada às propriedades redox do catalisador. Segundo Bozo et $a l,{ }^{11}$ que estudaram a participação do suporte de cério e zircônio em catalisadores de Pt e Pd na oxidação do metano, a atividade resulta da cooperação entre o metal, que é responsável pela dissociação do metano, e o suporte, que fornece espécies oxigênio ativo de sua estrutura. Eles destacam que a presença de vacâncias de oxigênio parece ser um fator chave na atividade, uma vez que permitem a transferência de elétrons para os metais nobres, além de promover a mobilidade e a labilidade dos oxigênios da estrutura do suporte de CZ. 
Novamente, observa-se um melhor desempenho por parte dos catalisadores envelhecidos a $900{ }^{\circ} \mathrm{C}$ em relação ao novo. Como na oxidação do $\mathrm{CO}$ no Pd-CZ, o impacto do tempo de envelhecimento a $900{ }^{\circ} \mathrm{C}$ praticamente foi desprezível.

Esse comportamento já foi observado em trabalhos anteriores, ${ }^{26-28}$ que o atribuíram às dimensões das partículas metálicas. Fan et al. ${ }^{27}$ por exemplo, testaram três catalisadores de Pt-CZ-La para as reações de oxidação do $\mathrm{CO}$ e propano e encontraram as menores atividades para o catalisador de maior dispersão metálica. Dessa forma, com o aumento do tamanho de partícula, planos cristalinos mais favoráveis à oxidação do propano seriam expostos, aumentando a taxa de reação. De maneira similar, Hicks et al. ${ }^{28}$ mediram as frequências de turnover para a oxidação completa do metano em catalisadores de Pt e Pd suportados em alumina, e encontraram as maiores TOF's para aqueles menos dispersos. Parece, portanto, existir um tamanho ótimo para os cristalitos metálicos serem mais ativos.

Neste trabalho, com base nos resultados de DRX notam-se indícios da presença de $\mathrm{PdO}$ principalmente nos catalisadores envelhecidos, que se acentua com o aumento da temperatura de envelhecimento. Isso indica que houve aglomeração dos cristais de $\mathrm{PdO}$, que se tornaram visíveis à difração de raios $\mathrm{X}$. No entanto, o aumento excessivo do cristalito é indesejável, pois pode reduzir por completo sua atividade, como após o envelhecimento a $1200^{\circ} \mathrm{C}$.

A partir dessa discussão, provavelmente, nos sistemas Pd-CZ a coincidência nas curvas de oxidação do $\mathrm{CO}$ obtidas para os catalisadores Pd-CZ novo e envelhecidos a $900{ }^{\circ} \mathrm{C}$ (Figura 7) se deve a dois aspectos: o ganho pela presença do metal e as perdas com o envelhecimento térmico a $900{ }^{\circ} \mathrm{C}$ que mascaram a ativação observada pelo suporte (Figura 7) devido ao impacto da temperatura nas partículas metálicas que se aglomeram, diminuindo o número de sítios ativos. No caso da oxidação do propano, a ativação a $900{ }^{\circ} \mathrm{C}$ é ainda nítida, com o catalisador novo apresentando um desempenho inferior aos encontrados após envelhecimento a $900{ }^{\circ} \mathrm{C}$.

\section{CONCLUSÕES}

O envelhecimento térmico promove a redutibilidade do sistema CZ como observado pela técnica de TPR, justificando o melhor desempenho catalítico na reação de oxidação do $\mathrm{CO}$ verificado para a maioria dos catalisadores da série $\mathrm{CZ}$, inclusive para os envelhecidos a $1200{ }^{\circ} \mathrm{C}$.

A impregnação de paládio no $\mathrm{CZ}$, gerando a série $\mathrm{Pd}-\mathrm{CZ}$, mostrou que esses catalisadores são mais ativos nas reações de oxidação do $\mathrm{CO}$ e do propano, porém, são aparentemente mais sensíveis ao envelhecimento térmico. Temperaturas de envelhecimento ao redor de 1200 ${ }^{\circ} \mathrm{C}$ por $12 \mathrm{~h}$ de exposição desativaram completamente o catalisador.

\section{MATERIAL SUPLEMENTAR}

Como material suplementar, disponível em http://quimicanova. sbq.org.br, incluem-se as Figuras $1 \mathrm{~S}$ e $2 \mathrm{~S}$, que complementam as Figuras 1 e 2 do artigo, pois se referem aos resultados obtidos para os catalisadores da série Pd-CZ.

\section{REFERÊNCIAS}

1. Bedrane, S.; Descorme, C.; Duprez, D.; Stud. Surf. Sci. Catal. 2001, 138, 125.

2. Nagai, Y.; Yamamoto, T.; Tanaka, T.; Yoshida, S.; Nonaka, T.; Okamoto, T.; Suda, A.; Sugiura, M.; Catal. Today 2002, 74, 225.

3. Boaro, M.; Vicario, M.; Leitenburg, C.; Dolcetti, G.; Trovarelli, A.; Catal. Today 2003, 77, 407.

4. Gandhi, H. S.; Graham, G. W.; Mccabe, R. W.; J. Catal. 2003, $216,433$.

5. Hungría, A. B.; Calvino, J. J.; Anderson, J. A.; Martinez-Árias, A.; Appl. Catal., B 2006, 62, 359.

6. Kubacha, A.; Martinez-Arias, A.; Fernández-Garcia, M.; Newton, M. A.; Catal. Today 2009, 145, 288.

7. Zotin, F. M. Z.; Gomes, O. F. M.; Alcover Neto, A.; Cardoso, M. B.; Catal. Today 2005, 107-108, 157.

8. Fernandes, D. M.; Alcover Neto, A.; Cardoso, M. J. B.; Zotin, F. M. Z.; Catal. Today 2008, 133-135, 574.

9. Fernandes, D. M.; Scofield, C. F.; Alcover Neto, A.; Cardoso, M. J. B.; Zotin, F. M. Z.; Process Saf. Environ. Prot. 2009, 87, 315.

10. Lassi, U.; Polvinen, R.; Kallinen, K.; Savimaki, A.; Appl. Catal., A 2004, 263, 241.

11. Bozo, C.; Gaillard, F.; Guilhaume, N.; Appl. Catal., A 2001, $220,69$.

12. Granados, M. L.; Galisteo, F. C.; Mariscal, R.; Alifanti M.; Gurbani, A.; Fierro, J. L. G.; Fernández-Ruíz, R.; Appl. Surf. Sci. 2006, 252, 8442 .

13. Hungría, A. B.; Browning, N. D.; Erni, R. P.; Fernández-Gárcia, M.; Conesa, J. C.; P’Rez Omil, J. A.; Martinéz-Árias, A.; J. Catal. 2005, 235, 251.

14. Kenevey, K.; Valdivieso, F.; Soustelle. M.; Pijolat, M.; Appl. Catal., B 2001, 29, 93.

15. Di Monte, R.; Fornasiero, P.; Kaspar, J.; Rumore, P.; Gubitosa, G.; Graziane, M.; Appl. Catal., B 2000, 24, 157.

16. Hori, C. E.; Ng, K. Y. S.; Brenner, A.; Rahmoeller, K. M.; Belton, D. N.; Braz. J. Chem. Eng. 2001, 18, 1.

17. Hori, C. E.; Permana, H.; Ng, K. Y. S.; Brenner, A.; More, K.; Rahmoeller, K. M.; Belton, D.; Appl. Catal., B 1998, 16, 105.

18. Sing, K. S. W.; Everett, D. H.; Haul, R. A. W.; Pierotti, R. A.; Rouquérol, J.; Siemieniewska, T.; Pure Appl. Chem. 1985, 57, 603.

19. Vidal, H.; Kaspar, J.; Pijolat, M.; Colon, G.; Bernal, S.; Cordón, A.; Perrichon, V.; Fally, F.; Appl. Catal., B 2000, 27, 49.

20. Wu, X.; Fan, J.; Ran, R.; Weng, D.; Chem. Eng. J. 2005, 109, 133.

21. Fornasiero, P.; Balducci, G.; Monte, R. Di.; Kaspar, J.; Sergo, V.; Gubitosa, G.; Ferrero, A.; Graziane, M.; J. Catal. 1996, 164, 173.

22. Fally, F.; Perrichon V.; Vidal, H.; Kaspar, J.; Blanco, G.; Pintado, J. M.; Bernal, S.; Colond, G.; Daturi, M.; Lavalley, J. C.; Catal. Today 2000, $59,373$.

23. Lambrou, P. S.; Costa, C. N.; Christou, S. Y.; Efstathiou, A. M.; Appl. Catal., B 2004, 54, 237.

24. Kaspar, J.; Fornasiero, P.; J. Solid State Chem. 2003, 171, 19.

25. Burch, R.; Crittle, D. J.; Hayes, M. J.; Catal. Today 1999, 47, 229.

26. Fernandes, D. M.; Dissertação de Mestrado, Universidade Federal do Rio de Janeiro, Brasil, 2007.

27. Fan, J.; Wu, X.; Yang, L.; Weng, D.; Catal. Today 2007, 126, 303.

28. Gélin, P.; Primet, M.; Appl. Catal., B 2002, 39, 1. 